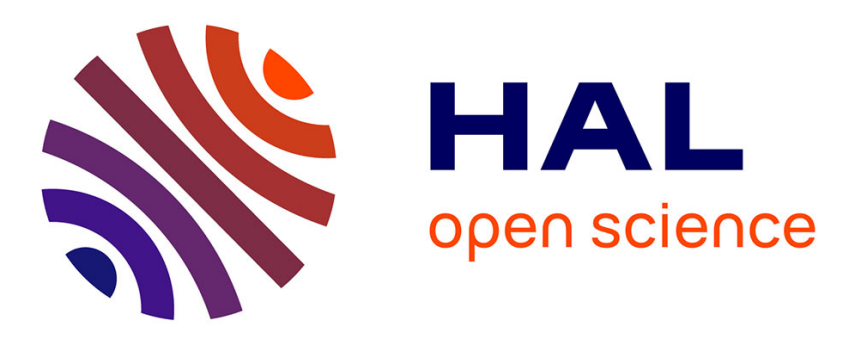

\title{
Influence of epitaxial strain on elastocaloric effect in ferroelectric thin films
}

Yang Liu, Wei Jie, Xiaojie Lou, Laurent Bellaiche, James F. Scott, Brahim Dkhil

\section{- To cite this version:}

Yang Liu, Wei Jie, Xiaojie Lou, Laurent Bellaiche, James F. Scott, et al.. Influence of epitaxial strain on elastocaloric effect in ferroelectric thin films. Applied Physics Letters, 2015, 106, pp.032901. 10.1063/1.4906198 . hal-01258503

\section{HAL Id: hal-01258503 https://hal.science/hal-01258503}

Submitted on 25 Aug 2020

HAL is a multi-disciplinary open access archive for the deposit and dissemination of scientific research documents, whether they are published or not. The documents may come from teaching and research institutions in France or abroad, or from public or private research centers.
L'archive ouverte pluridisciplinaire HAL, est destinée au dépôt et à la diffusion de documents scientifiques de niveau recherche, publiés ou non, émanant des établissements d'enseignement et de recherche français ou étrangers, des laboratoires publics ou privés. 


\section{Influence of epitaxial strain on elastocaloric effect in ferroelectric thin films}

Cite as: Appl. Phys. Lett. 106, 032901 (2015); https://doi.org/10.1063/1.4906198

Submitted: 17 December 2014 . Accepted: 06 January 2015. Published Online: 20 January 2015

Yang Liu, Jie Wei, Xiaojie Lou, L. Bellaiche, James F. Scott, and Brahim Dkhil
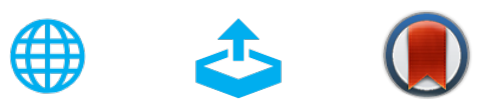

View Online

Export Citation

\section{ARTICLES YOU MAY BE INTERESTED IN}

Giant room-temperature barocaloric effect and pressure-mediated electrocaloric effect in $\mathrm{BaTiO}_{3}$ single crystal

Applied Physics Letters 104, 162904 (2014); https://doi.org/10.1063/1.4873162

Elastocaloric effect in ferroelectric ceramics

Applied Physics Letters 106, 172901 (2015); https://doi.org/10.1063/1.4919453

Direct and indirect measurements on electrocaloric effect: Recent developments and perspectives

Applied Physics Reviews 3, 031102 (2016); https://doi.org/10.1063/1.4958327

\section{Lock-in Amplifiers up to $600 \mathrm{MHz}$}
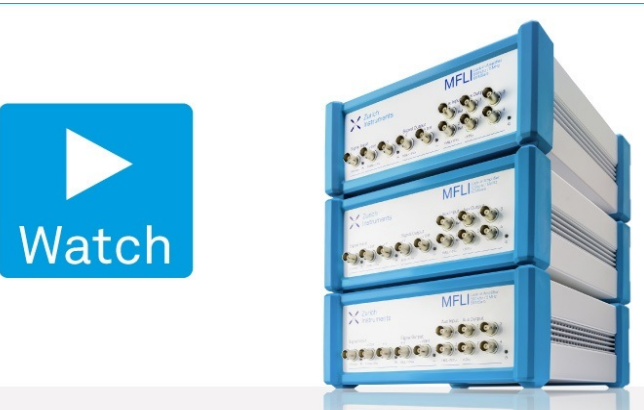


\title{
Influence of epitaxial strain on elastocaloric effect in ferroelectric thin films
}

\author{
Yang Liu, ${ }^{1, a)}$ Jie Wei, ${ }^{1,2}$ Xiaojie Lou, ${ }^{3}$ L. Bellaiche, ${ }^{4}$ James F. Scott, ${ }^{5}$ and Brahim Dkhil ${ }^{1}$ \\ ${ }^{1}$ Laboratoire Structures, Propriétés et Modélisation des Solides, UMR 8580 CNRS-CentraleSupélec, Grande \\ Voie des Vignes, Châtenay-Malabry Cedex 92295, France \\ ${ }^{2}$ Electronic Materials Research Laboratory, Key Laboratory of Ministry of Education, Xi' an Jiaotong \\ University, Xi' an 710049, People's Republic of China \\ ${ }^{3}$ Multi-disciplinary Materials Research Center, Frontier Institute of Science and Technology, and State \\ Key Laboratory for Mechanical Behavior of Materials, Xi' an Jiaotong University, Xi' an 710049, \\ People's Republic of China \\ ${ }^{4}$ Physics Department and Institute for Nanoscience and Engineering, University of Arkansas, Fayetteville, \\ Arkansas 72701 , USA \\ ${ }^{5}$ Department of Physics, Cavendish Laboratory, University of Cambridge, Cambridge CB3 OHE, \\ United Kingdom
}

(Received 17 December 2014; accepted 6 January 2015; published online 20 January 2015)

We report the influence of epitaxial strain $u_{m}$ on the elastocaloric properties of $\mathrm{BaTiO}_{3}$ thin films. Using thermodynamic calculations, we show that there exists a critical compressive stress $\sigma_{3 c}$ at which the elastocaloric effect is maximized for any compressive misfit strain we investigate. Moreover, it is found that $\left|\sigma_{3 c}\right|$ decreases significantly with decreasing $\left|u_{m}\right|$, which is accompanied by a reduction of the elastocaloric response. Interestingly, a several fold enhancement in the electrocaloric effect can be achieved for stress in proximity of $\sigma_{3 c}$. The elastocaloric effect predicted here may find potential cooling applications by combining the stress-mediated electrocaloric effect or designing hybrid elastocaloric/electrocaloric devices in the future. (C) 2015 AIP Publishing LLC. [http://dx.doi.org/10.1063/1.4906198]

The elastocaloric effect in solids, originally termed as piezocaloric effect, ${ }^{1}$ refers to the temperature (entropy) change in response to a uniaxial stress under adiabatic (isothermal) conditions. ${ }^{2,3}$ Research on this topic has aroused growing interest especially since the discovery of the giant elastocaloric effect in ferroelastic martensites. ${ }^{4}$ On one hand, ferroelastic shape memory alloys are most intensively studied and promising elastocaloric materials due to their excellent superelasticity and broad operational temperature window. ${ }^{5-9}$ On the other hand, ferroelastic ferroelectric bulk and thin films are predicted to have remarkable elastocaloric properties comparable with ferroelastic martensites, ${ }^{10-12}$ which further expands the elastocaloric family.

Indeed, issues of latent heat, size effect, and fatigue in ferroelastic elastocalorics have attracted more attention. ${ }^{13}$ For instance, it was reported that the hysteresis loss can be lowered in Fe-Pd single crystal exhibiting a second-order like martensitic transition. ${ }^{6}$ In addition, as the size of current shape-memory alloys shrinks towards the nano-scale, numerous problems and instabilities arise, including fatigue, micro-cracking, and oxidation. ${ }^{14}$ In this regard, ferroelectric thin films may have potential advantages. ${ }^{15}$ Interestingly, it was demonstrated very recently that a giant elastocaloric effect can be achieved in ferroelectric ultrathin films. ${ }^{12}$ Moreover, changing the mechanical boundary conditions by varying the epitaxial misfit strain can remarkably affect the phase transition, which may be helpful to tune the elastocaloric properties of ferroelectric thin films. ${ }^{16-19}$ This is of great interest and importance to design the elastocaloric cooling devices in the future and therefore motivates us to study the elastocaloric effect in ferroelectric thin films under the influence of epitaxial strain.

In this letter, we consider (001) $\mathrm{BaTiO}_{3}$ (BTO) films epitaxially grown on several compressive substrates, namely, $\mathrm{GdScO}_{3}$ (GSO), $\mathrm{DyScO}_{3}$ (DSO), $\mathrm{SrTiO}_{3}$ (STO), and $\mathrm{NdGaO}_{3}$ (NGO), having various in-plane misfit strains $u_{m}$ ranging from $-1 \%$ for $\mathrm{GSO}^{17}$ to $-3.2 \%$ for $\mathrm{NGO}^{20}$ owing to mismatch in lattice parameters between the films and the underlying substrates. We restrict ourselves to the case of a uniform local loading $\sigma_{3}$ applied along the $\mathrm{z}$ direction (perpendicular to the film surface). We neglect the shear stresses $\sigma_{4}$ and $\sigma_{5}$ and the flexoelectric effect caused by strain gradients ${ }^{21-23}$ to ensure that the effect of epitaxial strain on elastocaloric properties is clearly observable from the calculations. ${ }^{12,24}$ Compressive substrates are chosen, since we only consider the polarization and strain change along the direction $\mathrm{z}$ (out-of plane) conjugate to the direction of the external stress according to the "misfit strain-stress" phase diagrams. ${ }^{24}$ Therefore, the transition from the paraelectric phase $\left(P_{x}=P_{y}=P_{z}=0\right)$ to ferroelectric tetragonal phase $\left(P_{x}=P_{y}=0, P=P_{z} \neq 0\right)$ is favored to occur near room temperature. ${ }^{24}$ Note that the maximum $\sigma_{3}$ we used is well below the local stress threshold $(\sim 20 \mathrm{GPa})$ for irreversible plastic damage of the BTO surface. ${ }^{25}$ Taking into account the mechanical boundary conditions, the free energy can be expressed as ${ }^{24}$

$$
\begin{aligned}
F= & \frac{1}{2} \alpha_{1}^{*} P^{2}+\frac{1}{4} \alpha_{11}^{*} P^{4}+\frac{1}{6} \alpha_{111} P^{6}+\frac{1}{8} \alpha_{1111} P^{8} \\
& -\frac{1}{2} S_{11} \sigma_{3}^{2}+\frac{\left(u_{m}-S_{12} \sigma_{3}\right)^{2}}{S_{11}+S_{12}}-E P,
\end{aligned}
$$

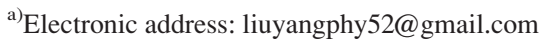

where 


$$
\begin{gathered}
\alpha_{1}^{*}=\alpha_{1}-\frac{4 Q_{12}}{S_{11}+S_{12}} u_{m}-2\left(Q_{11}-\frac{2 Q_{12} S_{12}}{S_{11}+S_{12}}\right) \sigma_{3} \\
\alpha_{11}^{*}=\alpha_{11}+\frac{4 Q_{12}^{2}}{S_{11}+S_{12}}
\end{gathered}
$$

are the renormalized Landau coefficients. $\alpha_{i j k l}$ are the dielectric stiffness coefficients at constant stress, and only $\alpha_{1}$ is temperature dependent through the Curie-Weiss law $\alpha_{1}=\left(T-T_{0}\right) / \varepsilon_{0} C$, $T_{0}$ and $C$ being the Curie-Weiss temperature and constant, respectively. $S_{m n}$ and $Q_{m n}$ are the elastic compliances and the electrostrictive coefficients of the films, respectively. $E$ is the external electric field which is applied along the polar axis.

The equilibrium strain $u_{3}$ and polarization $P_{0}$ can be derived from the condition of thermodynamic equilibrium: $\partial F / \partial \sigma_{3}=-u_{3}, \partial F / \partial P=0$. As a result, we have

$$
\begin{aligned}
u_{3}= & \left(S_{11}-\frac{2 S_{12}^{2}}{S_{11}+S_{12}}\right) \sigma_{3}+\frac{2 S_{12}}{S_{11}+S_{12}} u_{m} \\
& -\left(Q_{11}-\frac{2 S_{12} Q_{12}}{S_{11}+S_{12}}\right) P_{0}^{2} .
\end{aligned}
$$

It can be seen clearly that only the last term depending on $P_{0}^{2}$ in Eq. (4) contributes to the elastocaloric effect due to its temperature dependence (since it is straightforward to prove that $P_{0}$ depends on $\alpha_{1}$ ).

The elastocaloric change in temperature $\Delta T_{\sigma_{3}}$ upon application of a stress field in adiabatic conditions can be determined by ${ }^{5,12}$

$$
\Delta T_{\sigma_{3}}=-\int_{0}^{\sigma_{3}} \frac{T}{C_{\sigma_{3}}}\left(\frac{\partial u_{3}}{\partial T}\right)_{\sigma_{3}, E} d \sigma_{3},
$$

where the Maxwell relation $\left(\frac{\partial S}{\partial \sigma_{3}}\right)_{E, T}=\left(\frac{\partial u_{3}}{\partial T}\right)_{\sigma_{3}, E}$ is used. $C_{\sigma_{3}}$ is the heat capacity under constant stress $\sigma_{3}$. Note that in order to ensure that the effect of misfit strain on the elastocaloric response is clearly seen from our thermodynamical calculations, we simplify by neglecting the depolarizing field and the substrate deformations. ${ }^{12}$
From the viewpoint of practical applications, it is of interest to set the initial temperature $T$ at room temperature $(300 \mathrm{~K})$. All the parameters we used are the same as those in Ref. 26. Figs. 1(a)-1(d) summarize the stress-dependent equilibrium polarization $P_{0}$, strain $u_{3}$, and adiabatic temperature change $\Delta T_{\sigma_{3}}$, respectively. It is shown in Fig. 1(a) that there exists a critical stress field $\sigma_{3 c}$ at which $P_{0}$ continuously disappears, which is essentially attributed to the secondorder phase transition $\left(\alpha_{11}^{*}>0\right)$ modified by the mechanical boundary conditions. ${ }^{24}$ As the misfit-strain $\left|u_{m}\right|$ increases, $\left|\sigma_{3 c}\right|$ increases remarkably from $2.97 \mathrm{GPa}$ for GSO to 8.30 GPa for NGO, which indicates that polarization in BTO thin films grown on NGO substrate is more difficult to suppress by a uniaxial stress than the other substrates. And the total polarization change induced by different $\sigma_{3 c}$ increases considerably as $\left|u_{m}\right|$ increases.

Fig. 1(b) shows that a large strain change can be achieved, which is in line with our previous result. ${ }^{12}$ For instance, for the film on GSO substrate, the strain change is about $1.6 \%$ under a compressive stress of $-3 \mathrm{GPa}$, which is approximately two times as large as those reported in superelastic BTO single crystal either $(0.75 \%)$ induced by a low electric field of $2 \mathrm{kV} / \mathrm{cm}$ (Ref. 27 ) or $(0.85 \%)$ by a modest stress field of $15 \mathrm{MPa}$ (Ref. 28).

As expected, the noticeable elastocaloric effect is depicted in Figs. 1(c) and 1(d). It appears that the elastocaloric response of BTO thin films is maximized when the stress reaches $\sigma_{3 c}$. After that, no additional change in $\Delta T_{\sigma_{3}}$ is observed due to the disappearance of the polarization (see Fig. 1(a), Eqs. (4) and (5)). $\left|\Delta T_{\sigma_{3}}\right|$ increases as $\left|u_{m}\right|$ increases and reaches its largest value of $6.9 \mathrm{~K}$ at $8.30 \mathrm{GPa}$ (on NGO substrate). Note that $6.9 \mathrm{~K}$ is indeed in the range of temperature changes for other elastocaloric systems reported in the literature. $^{2-11}$ Moreover, due to the second-order nature of the phase transition, it is expected that the hysteresis loss and irreversibilities associated with latent heat vanish, which is of importance to enhance refrigerant efficiency. ${ }^{5,12,13}$ In addition, the system can operate in a wide temperature window for all compressive substrates we investigated (see, e.g., the $60 \mathrm{~K}$
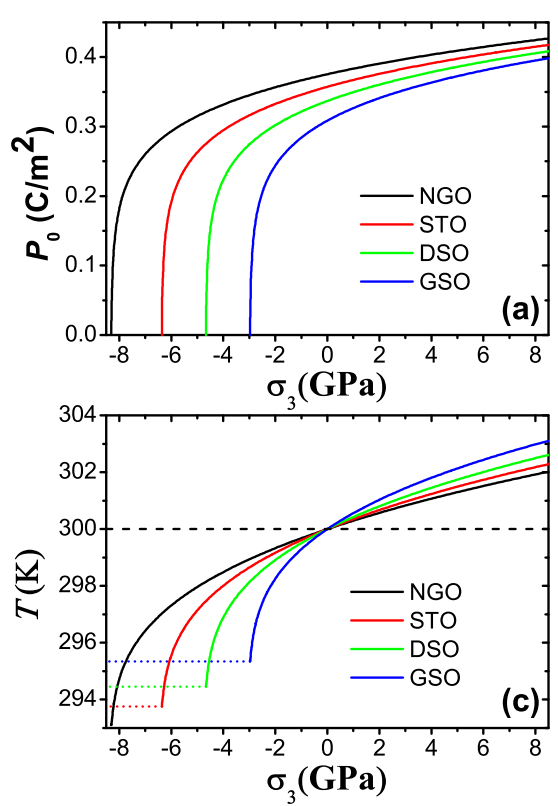
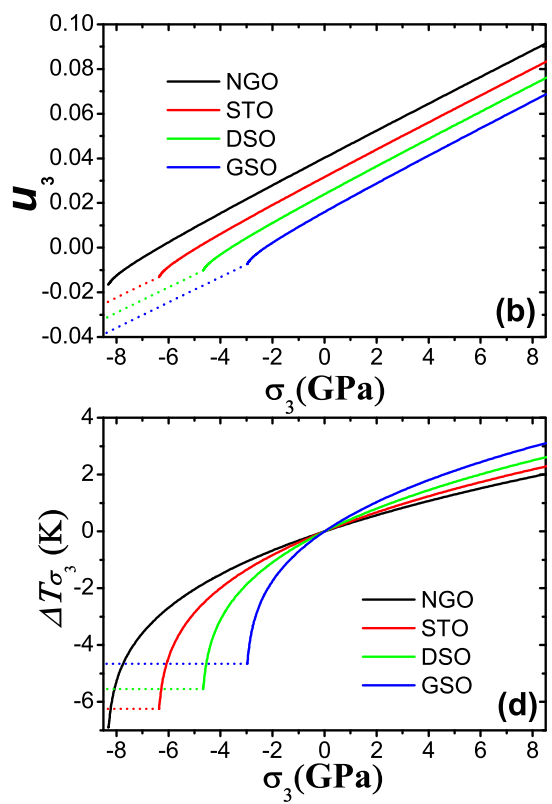

FIG. 1. The dependence of (a) polarization $P_{0}$, (b) strain $u_{3}$, (c) sample temperature $T$, and (d) adiabatic temperature change $\Delta T_{\sigma_{3}}$ on stress $\sigma_{3}$ in epitaxial BTO thin films grown on different compressive substrates at room temperature. The dashed line in (c) indicates the initial temperature which is set as room temperature $(300 \mathrm{~K})$. 

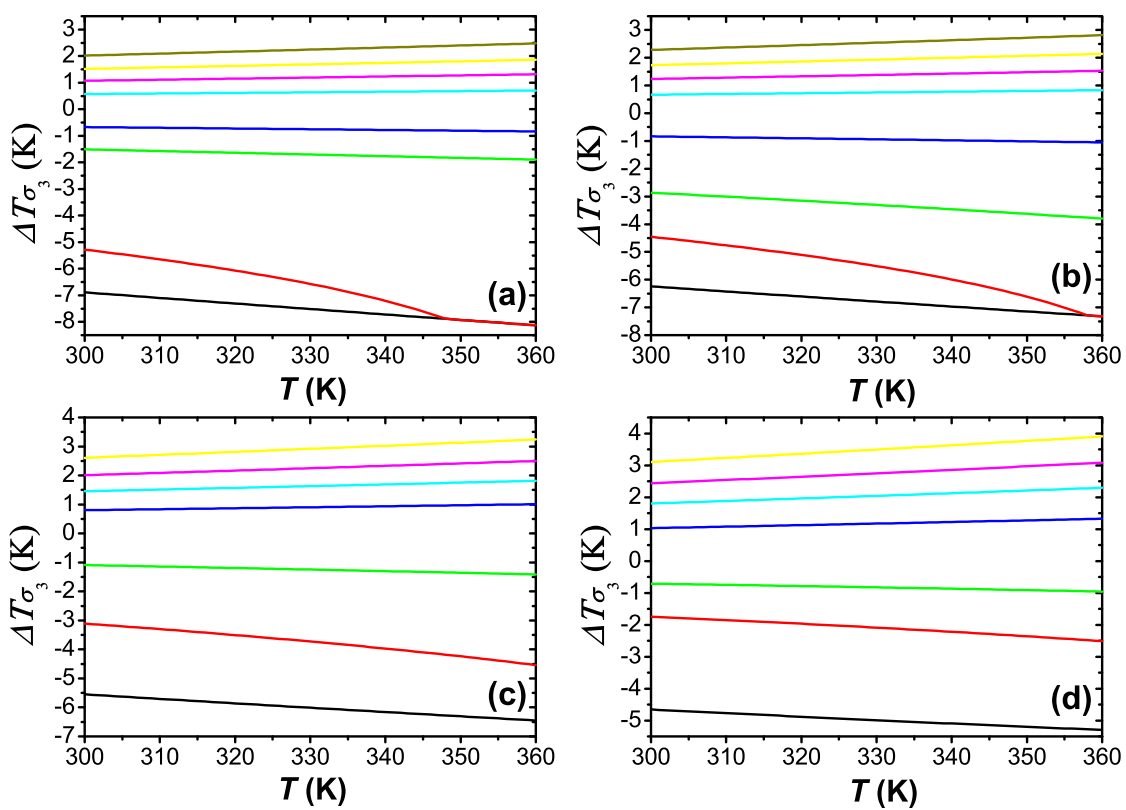

FIG. 2. Dependence of $\Delta T_{\sigma_{3}}$ on $T$ of epitaxial BTO thin films grown on (a) NGO, (b) STO, (c) DGO, and (d) GSO substrates. The different curves correspond to different stress values: from top to bottom the curves correspond to (a) $8.5,6,4,2,-2,-4,-8$, and $-8.5 \mathrm{GPa}$; (b) $8.5,6,4,2,-2,-5,-6$, and $-6.5 \mathrm{GPa}$; (c) $8.5,6,4,2,-2,-4$, and $-5 \mathrm{GPa}$; (d) $8.5,6,4,2,-1,-2$, and $-3 \mathrm{GPa}$ window considered in Figs. 2(a)-2(d)), which is very desired for cooling applications. Therefore, here we demonstrate that ferroelectric thin films may be good candidates for elastocaloric cooling, which also can potentially expand the elastocaloric family from macroscopic scale to nanoscale. ${ }^{12}$

The foregoing findings are summarized in Fig. 3. It is shown that the critical driving stress $\left|\sigma_{3 c}\right|$ decreases significantly from $8.30 \mathrm{GPa}$ for NGO to $2.97 \mathrm{GPa}$ for GSO as misfitstrain $\left|u_{m}\right|$ decreases, which can provide an effective approach to lower the driving stress. However, the elastocaloric peak $\Delta T_{\sigma_{3}}(\max )$ also experiences a considerable decrease from $6.9 \mathrm{~K}$ to $4.6 \mathrm{~K}$ during that change in $\left|u_{m}\right|$. Note that the relevant figure of merit for real devices depends on the external force rather than the applied stress. Though a much higher driving stress is required here, which is orders-of-magnitude larger than those reported in other typical elastocaloric materials, ${ }^{4-11}$ only an extremely small force is needed for ferroelectric thin films. Fig. 3 clearly shows that the elastocaloric response of ferroelectric thin films can be remarkably tuned

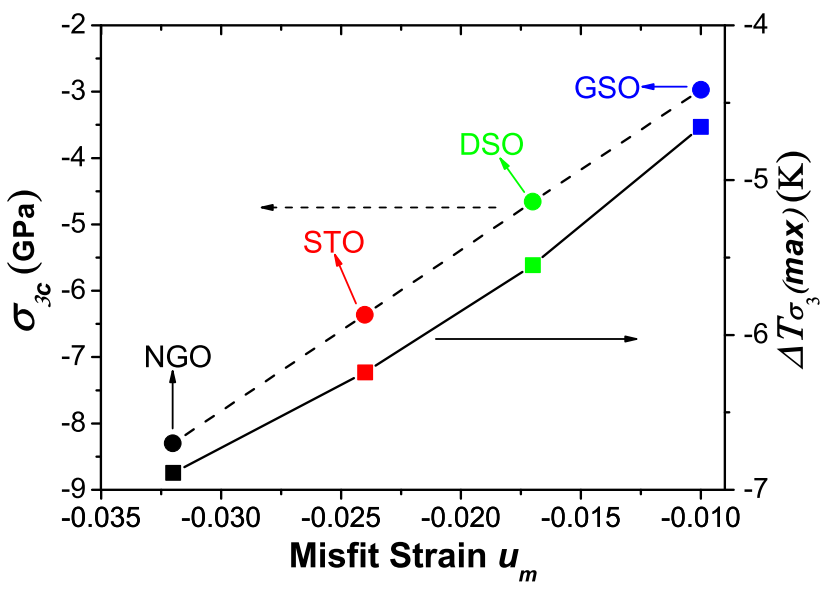

FIG. 3. The critical driving stress $\sigma_{3 c}$ and corresponding maximum elastocaloric response $\Delta T_{\sigma_{3}}(\max )$ in epitaxial BTO thin films grown on different compressive substrates at room temperature. by changing the substrates. Interestingly, if the substrate could be electrically active, designing hybrid elastocaloric/electrocaloric devices controlled by an electric field rather than a mechanical stress can be considered in the future.

Note that we assume that thermal expansion coefficients (TECs) in the paraelectric phase play a negligible contribution to the corresponding entropy change. Indeed, this nominal TEC behavior is directly supported by recent theoretical and experimental results in various materials. For instance, a direct approach based on first-principles calculations does show that the elastocaloric response in $\mathrm{PbTiO}_{3}$ above the phase transition temperature is nearly zero, while it only emerges through by the appearance of the polarization induced by a large enough tensile stress (see Fig. 2 in Ref. 11 for more details). Immediately, one can see that the TECs are negligible in the paraelectric phase according to Eqs. (4) and (5). This behavior is also confirmed by a recent thermodynamic model showing that both the conventional elastocaloric response ${ }^{29}$ and inverse barocaloric effect ${ }^{30}$ in BTO single crystals disappear in the paraelectric phase. On the other hand, it was reported in both ferroelectric $\mathrm{PbTiO}_{3}{ }^{31}$ and ferromagnetic $\mathrm{La}-\mathrm{Fe}-\mathrm{Co}-\mathrm{Si}^{32}$ that the appreciable barocaloric effect can be only experimentally observed in vicinity of the phase transition temperature. Indeed, both the elastocaloric and barocaloric response strongly depend on the relationship of TEC versus temperature. Since the TEC peaks at the phase transition and the TEC versus temperature curves are almost flat in the paraelectric phase, the corresponding intrinsic entropy change in paraelectric phase cannot be comparable with that near the phase transition. ${ }^{31,32}$ Therefore, it is rational to ignore the contribution from that above the phase transition. In this regard, the corresponding TEC can therefore be set to be extremely small or zero in the paraelectric phase. Most importantly, we found that the TECs experienced a dramatic change by one order of magnitude for a stress reaching the critical stress, ${ }^{12}$ which strongly supports that our foregoing treatment is reasonable. 

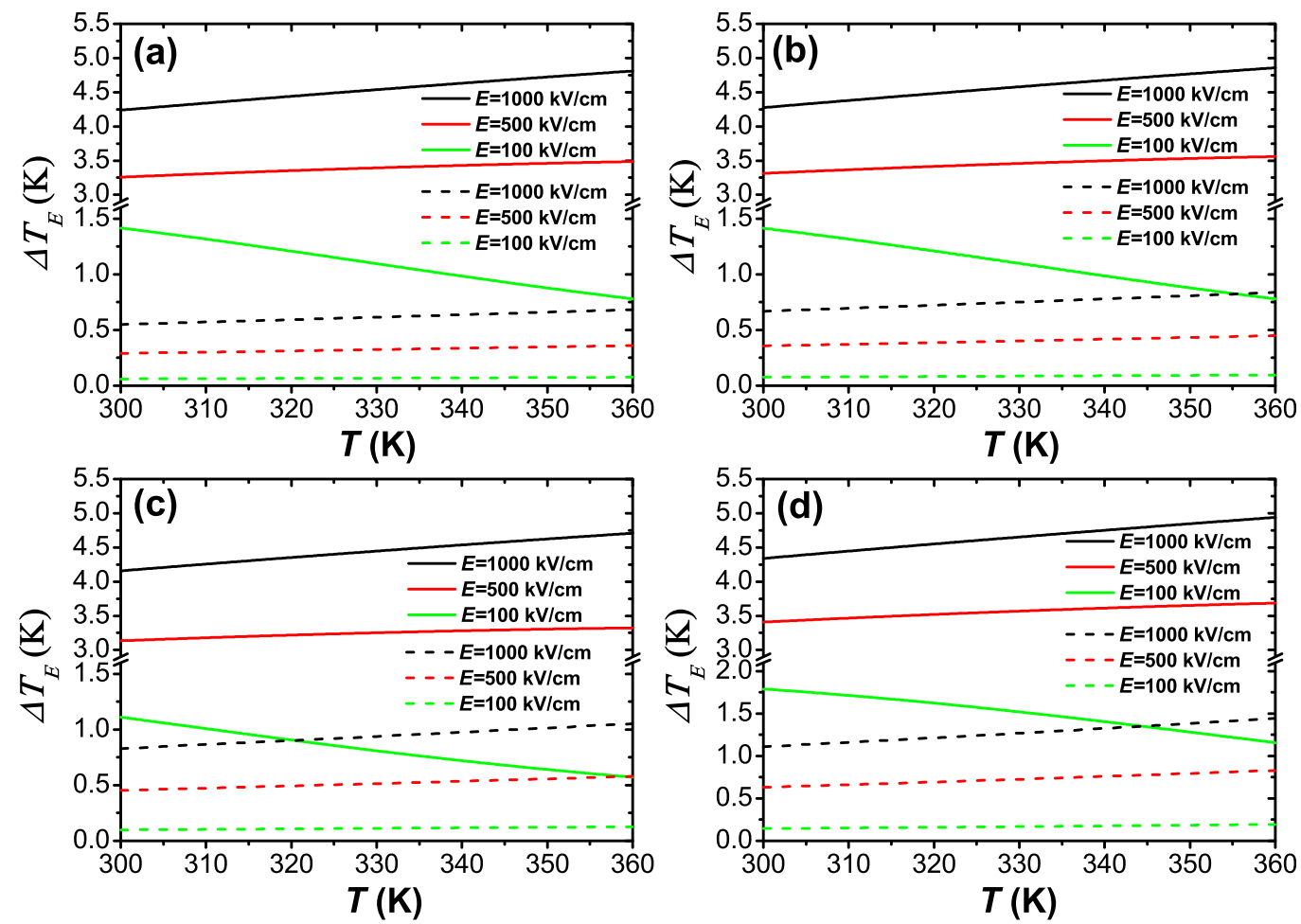

FIG. 4. Electrocaloric effect in epitaxial BTO thin films under different compressive loadings (solid line): (a) on a NGO substrate $\left(\sigma_{3}=-8.5 \mathrm{GPa}\right)$, (b) on a STO substrate $\left(\sigma_{3}=-6.5 \mathrm{GPa}\right)$, (c) on a DGO substrate $\left(\sigma_{3}=-5 \mathrm{GPa}\right)$ and on a GSO substrate $\left(\sigma_{3}=-3 \mathrm{GPa}\right)$, respectively. The dashed line refers to the electrocaloric effect in the unloaded state.

The TECs we calculated here may be called as anomalous ones while the normal contributions are presumably set to be negligible (i.e., zero in our case) compared to anomalous parts. The normal TEC can be added by introducing the thermo-electric and the thermo-elastic couplings into the free energy. It was reported that such modification allows to describe the evolution of polarization with temperature in the different polar phases in a slightly more accurate manner. ${ }^{33}$ This treatment may also result in non-zero TEC (normal TEC) in both ferroelectric and paraelectric phases. However, it is expected that the TECs in the paraelectric phase resulting from this modification should be almost flat or only weakly dependent on temperature, as evidenced by existing experiments. ${ }^{31,32}$ Obviously, the corresponding contribution to the entropy change induced by a stress field or a hydrostatic pressure in the paraelectric phase is still significantly smaller than that at the phase transition. Note that the anomalous part we concentrated on is much larger than the ordinary linear thermal expansion above the phase transition temperature as demonstrated recently by experiments in $\left(\mathrm{CH}_{3} \mathrm{NHCH}_{2} \mathrm{COOH}\right)_{3} \mathrm{CaCl}_{2}$ (TSCC). ${ }^{34}$ More importantly, it was shown by Lashley et al that the thermal expansion behavior does not satisfy Landau theory. Instead, it satisfies the defect-driven theory of Levanyuk and Sigov. ${ }^{35}$ Therefore, it is trivial to introduce such a risky term into free energy, not to mention that our results are already well supported by recent experimental and theoretical works.

Let us consider now the electrocaloric response which can be added to the elastocaloric one. Electrocaloric effect refers to the reversible temperature change $\Delta T_{E}$ upon adiabatic application or removal of an electric field. ${ }^{36-39}$ In particular, the electrocaloric properties of BTO thin films have been intensively investigated. ${ }^{12,18,19,26,40-47}$ Interestingly, it was reported that applying a uniaxial compressive stress could enhance and widen the electrocaloric response considerably in ultrathin BTO films due to tuning of the depolarization field. ${ }^{26}$ It is also reported that the electrocaloric peak under tensile stresses moves towards higher temperatures with its magnitude slightly enhanced in BTO single crystals. ${ }^{29}$ For the case under hydrostatic pressure, a slight reduction of the electrocaloric peak with increasing pressure is found in BTO single crystals. ${ }^{30} \Delta T_{E}$ can be determined as

$$
\Delta T_{E}=-\int_{0}^{E} \frac{T}{C_{E}}\left(\frac{\partial P_{0}}{\partial T}\right)_{E, \sigma_{3}} d E
$$

where $C_{E}$ is the heat capacity under constant electric field. For the unloaded case, it can be seen that as $\left|u_{m}\right|$ decreases, the electrocaloric response increases considerably, which agrees well with the previous study. ${ }^{19}$ Because the ferroelectricity in BTO thin films grown on NGO substrate is already larger than those grown on other substrates ${ }^{20}$ in this case, it is more difficult to achieve a larger polarization change under the same electric field. Indeed, mechanical stress may find use in tuning the electrocaloric effect. Stress may modify the height, the width, and the position of the peak in $\Delta T_{E}$ in ferroelectric thin films, according to our recent study. ${ }^{26}$

Here, we may consider the critical case as follows and we find that stress can act as a way to significantly enhance the electrocaloric effect, which is shown in Figs. 4(a)-4(d). For instance, it is shown in Fig. 4(a) that $\Delta T_{E}$ in the compressive loaded state $\left(\sigma_{3} \leq \sigma_{3 c}\right)$ is about $4.23 \mathrm{~K}(E=1000 \mathrm{kV} / \mathrm{cm})$ at $300 \mathrm{~K}$ which is over 6 times larger than that $(0.55 \mathrm{~K})$ in the unloaded case at $300 \mathrm{~K}$ under the same $E$ (NGO substrate). 
Note that the polarization is close to zero when the stress is in proximity of $\sigma_{3 c}$. Therefore, even a modest electric field can readily polarize the film and produce a remarkably enhanced electrocaloric response. As a result, it can be seen from Figs. 4(a)-4(d) that a several fold enhancement in the electrocaloric effect can be achieved. This behavior is observed within the temperature range of interest and for all substrates we considered (see Figs. 4(a)-4(d)). Therefore, our findings in this work may also open a route for cooling applications by combining the enhanced electrocaloric effect mediated by mechanical stress and elastocaloric effect in a single material. ${ }^{12,26}$ Note that a very recent study reported that secondary contribution from the piezoelectric strain and associated elastocaloric effect in $\mathrm{PbZr}_{1-x} \mathrm{Ti}_{x} \mathrm{O}_{3}$ thin films may reduce the overall electrocaloric entropy change. ${ }^{48}$ This secondary effect is expected to play a negligible role in our case since fully strained BTO thin films considered here show extremely small piezoelectric response. ${ }^{20}$

In summary, using a thermodynamic model we have studied the elastocaloric effect and stress-mediated electrocaloric effect in BTO thin films grown on different compressive substrates. Our results reveal that epitaxial strain can significantly tune the elastocaloric peak and the critical driving stress. It is shown that a several fold enhancement of the electrocaloric effect can be achieved for stress approaching to the critical value. Our preliminary findings suggest a potential route to design hybrid elastocaloric/electrocaloric devices controlled by a modest electric field and combine the elastocaloric effect and stress-mediated-and-enhanced electrocaloric effect in the same material.

Y.L., J.W., and B.D. thank the China Scholarship Council (CSC). X.J.L., J.W., Y.L., and B.D. thank the National Science Foundation of China (NSFC Nos. 51272204 and 51372195), the Ministry of Science and Technology of China through a 973-Project (No. 2012CB619401), and "One Thousand Youth Talents" program for support. L.B. thank NSF Grant No. DMR-1066158.

${ }^{1}$ J. F. Nye, Physical Properties of Crystals (Clarendon Press, Oxford, 1957), Chap. X.

${ }^{2}$ S. A. Nikitin, G. Myalikgulyev, M. P. Annaorazov, A. L. Tyurin, R. W. Myndyev, and S. A. Akopyan, Phys. Lett. A 171, 234 (1992).

${ }^{3}$ M. P. Annaorazov, S. A. Nikitin, A. L. Tyurin, K. A. Asatryan, and A. Kh. Dovletov, J. Appl. Phys. 79, 1689 (1996).

${ }^{4}$ E. Bonnot, R. Romero, L. Mañosa, E. Vives, and A. Planes, Phys. Rev. Lett. 100, 125901 (2008)

${ }^{5}$ L. Mañosa, A. Planes, and M. Acetb, J. Mater. Chem. A 1, 4925 (2013).

${ }^{6}$ F. Xiao, T. Fukuda, and T. Kakeshita, Appl. Phys. Lett. 102, 161914 (2013).

${ }^{7}$ J. Cui, Y. Wu, J. Muehlbauer, Y. Hwang, R. Radermacher, S. Fackler, M. Wuttig, and I. Takeuchi, Appl. Phys. Lett. 101, 073904 (2012).

${ }^{8}$ C. Bechtold, C. Chluba, R. Lima de Miranda, and E. Quandt, Appl. Phys. Lett. 101, 091903 (2012).

${ }^{9}$ L. Mañosa, S. Jarque-Farnos, E. Vives, and A. Planes, Appl. Phys. Lett. 103, 211904 (2013).

${ }^{10}$ S. Lisenkov and I. Ponomareva, Phys. Rev. B 86, 104103 (2012).

${ }^{11}$ S. Lisenkov, B. K. Mani, C.-M. Chang, J. Almand, and I. Ponomareva, Phys. Rev. B 87, 224101 (2013).

${ }^{12}$ Y. Liu, I. C. Infante, X. J. Lou, L. Bellaiche, J. F. Scott, and B. Dkhil, Adv. Mater. 26, 6132 (2014).

${ }^{13}$ S. Fähler, U. K. Rößler, O. Kastner, J. Eckert, G. Eggeler, H. Emmerich, P. Entel, S. Müller, E. Quandt, and K. Albe, Adv. Eng. Mater. 14, 10 (2012).
${ }^{14}$ T. Waitz, K. Tsuchiya, T. Antretter, and F. D. Fischer, MRS Bull 34, 814 (2009).

${ }^{15}$ J. X. Zhang, X. X. Ke, G. Y. Gou, J. Seidel, B. Xiang, P. Yu, W.-I. Liang, A. M. Minor, Y.-H. Chu, G. Van Tendeloo, X. B. Ren, and R. Ramesh, Nat. Commun. 4, 2768 (2013).

${ }^{16}$ N. A. Pertsev, A. G. Zembilgotov, and A. K. Tagantsev, Phys. Rev. Lett. 80, 1988 (1998); S. P. Alpay, I. B. Misirlioglu, A. Sharma, and Z.-G. Ban, J. Appl. Phys. 95, 8118 (2004).

${ }^{17}$ K. J. Choi, M. Biegalski, Y. L. Li, A. Sharan, J. Schubert, R. Uecker, P. Reiche, Y. B. Chen, X. Q. Pan, V. Gopalan, L.-Q. Chen, D. G. Schlom, and C. B. Eom, Science 306, 1005 (2004).

${ }^{18}$ G. Akcay, S. P. Alpay, J. V. Mantese, and G. A. Rossetti, Jr., Appl. Phys. Lett. 90, 252909 (2007)

${ }^{19}$ G. Akcay, S. P. Alpay, G. A. Rossetti, Jr., and J. F. Scott, J. Appl. Phys. 103, 024104 (2008).

${ }^{20}$ V. Garcia, S. Fusil, K. Bouzehouane, S. Enouz-Vedrenne, N. D. Marthur, A. Barthélémy, and M. Bibes, Nature 460, 81 (2009).

${ }^{21}$ A. K. Tagantsev, Phys. Rev. B 34, 5883 (1986).

${ }^{22} \mathrm{H}$. Lu, C.-W. Bark, D. Esque de los Ojos, J. Alcala, C. B. Eom, G. Catalan, and A. Gruverman, Science 336, 59 (2012).

${ }^{23}$ J. F. Scott, J. Phys.: Condens. Matter 25, 331001 (2013).

${ }^{24}$ A. Yu. Emelyanov, N. A. Pertsev, and A. L. Kholkin, Phys. Rev. B 66, 214108 (2002).

${ }^{25}$ Y. Gaillard, A. Hurtado Macías, J. Muñoz-Saldaña, M. Anglada, and G. Trápaga, J. Phys. D: Appl. Phys. 42, 085502 (2009).

${ }^{26}$ Y. Liu, I. C. Infante, X. J. Lou, D. C. Lupascu, and B. Dkhil, Appl. Phys. Lett. 104, 012907 (2014).

${ }^{27}$ X. B. Ren, Nat. Mater. 3, 91 (2004).

${ }^{28}$ Y. W. Li, X. B. Ren, F. X. Li, H. S. Luo, and D. N. Fang, Appl. Phys. Lett. 102, 092905 (2013).

${ }^{29}$ Y. Liu, J. Wei, P.-E. Janolin, I. C. Infante, J. Kreisel, X. J. Lou, and B. Dkhil, Phys. Rev. B 90, 104107 (2014).

${ }^{30}$ Y. Liu, J. Wei, P.-E. Janolin, I. C. Infante, X. J. Lou, and B. Dkhil, Appl. Phys. Lett. 104, 162904 (2014).

${ }^{31}$ E. A. Mikhaleva, I. N. Flerov, M. V. Gorev, M. S. Molokeev, A. V. Cherepakhin, A. V. Kartashev, N. V. Mikhashenok, and K. A. Sablina, Phys. Solid State 54, 1832 (2012).

${ }^{32}$ L. Mañosa, D. Gonzalez-Alonso, A. Planes, M. Barrio, J.-L. Tamarit, I. S. Titov, M. Acet, A. Bhattacharyya, and S. Majumdar, Nat. Commun. 2, 595 (2011).

${ }^{33}$ R. Renoud, M. Ragheb, C. Borderon, and H. W. Gundel, "Free energy of $\mathrm{BaTiO}_{3}$ single crystal," in Applications of Ferroelectric and Workshop on the Piezoresponse Force Microscopy (ISAF/PFM), Prague (2013), pp. 34-37.

${ }^{34}$ J. C. Lashley, J. H. D. Munns, M. Echizen, M. N. Ali, S. E. Rowley, and J. F. Scott, Adv. Mater. 26, 3860 (2014).

${ }^{35}$ J. F. Scott, EPL 104, 36004 (2013).

${ }^{36}$ A. S. Mischenko, Q. Zhang, J. F. Scott, R. W. Whatmore, and N. D. Mathur, Science 311, 1270 (2006).

${ }^{37}$ B. Neese, B. Chu, S. G. Lu, Y. Wang, E. Furman, and Q. M. Zhang, Science 321, 821 (2008).

${ }^{38}$ J. F. Scott, Annu. Rev. Mater. Res. 41, 229 (2011).

${ }^{39}$ Z. D. Luo, D.-W. Zhang, Y. Liu, D. Zhou, Y. G. Yao, C. Q. Liu, B. Dkhil, X. B. Ren, and X. J. Lou, Appl. Phys. Lett. 105, 102904 (2014).

${ }^{40}$ Y. Bai, G. P. Zheng, and S. Q. Shi, Appl. Phys. Lett. 96, 192902 (2010).

${ }^{41}$ S. Kar-Narayana and N. D. Mathur, J. Phys. D: Appl. Phys. 43, 032002 (2010).

${ }^{42}$ Y. Bai, G.-P. Zheng, K. Ding, L. J. Qiao, S.-Q. Shi, and D. Guo, J. Appl. Phys. 110, 094103 (2011).

${ }^{43}$ X. Zhang, J. B. Wang, B. Li, X. L. Zhong, X. J. Lou, and Y. C. Zhou, J. Appl. Phys. 109, 126102 (2011).

${ }^{44}$ Y. Liu, X.-P. Peng, X. J. Lou, and H. Zhou, Appl. Phys. Lett. 100, 192902 (2012).

${ }^{45}$ S. Kar-Narayan, S. Crossley, X. Moya, V. Kovacova, J. Abergel, A. Bontempi, N. Baier, E. Defay, and N. D. Mathur, Appl. Phys. Lett. 102, 032903 (2013).

${ }^{46}$ Y. Liu, X. J. Lou, M. Bibes, and B. Dkhil, Phys. Rev. B 88, 024106 (2013).

${ }^{47}$ Y. Liu, I. C. Infante, X. J. Lou, and B. Dkhil, Appl. Phys. Lett. 104, 082901 (2014).

${ }^{48}$ T. Tong, J. Karthik, R. V. K. Mangalam, L. W. Martin, and D. G. Cahill, Phys. Rev. B 90, 094116 (2014). 